\title{
Effect of circadian phase on context and cued fear conditioning in $\mathrm{C} 57 \mathrm{BL} / 6 \mathrm{~J}$ mice
}

\author{
VERÓNICA S. VALENTINUZZI \\ Northwestern University, Evanston, Illinois \\ and Universidade Estadual de Campinas, São Paulo, Brazil \\ DANIEL E. KOLKER and MARTHA HOTZ VITATERNA \\ Northwestern University, Evanston, Illinois \\ ELENICE A. M. FERRARI \\ Universidade Estadual de Campinas, São Paulo, Brazil \\ JOSEPH S. TAKAHASHI \\ Howard Huges Medical Institute, Chevy Chase, Maryland \\ and Northwestern University, Evanston, Illinois \\ and \\ FRED W. TUREK \\ Northwestern University, Evanston, Illinois
}

\begin{abstract}
We examined context-dependent and tone-cued fear conditioning during the activity and rest periods of C57BL/6J mice. Wheel-running activity was measured continuously as a marker of circadian phase. To control the effects of light on the response, the animals were kept in a skeleton photoperiod (two 15-min light pulses per day, indicating the beginning and the end of the day). Half the animals were trained and tested for context-dependent fear conditioning $2 \mathrm{~h}$ after the morning light pulse; the other half were trained and tested $2 \mathrm{~h}$ after the evening light pulse. Animals were tested every $24 \mathrm{~h}$ for 5 days to analyze the conditioned response and the rate of extinction. They were then trained for tonecued fear conditioning at the same time and tested for 5 consecutive days. A significant difference between the morning and the evening groups was observed in the conditioning level and extinction rate of context-dependent fear conditioning, but not in tone-cued fear conditioning. These results suggest a modulating effect of the biological clock on the context fear-conditioning pathway.
\end{abstract}

Rhythmicity has been reported in numerous biochemical, physiological, and behavioral systems. These rhythms reflect the combined influences of an endogenous biological clock and a range of exogenous influences (Turek, 1998). Many processes that influence memory display circadian oscillations, including brain gene expression (Holmes, French, \& Seckl, 1995; Menegazzi, Carcereri, De Prati, \& Zucconi, 1994), brain protein levels (Kononen, Koistinaho, \& Alho, 1990), neurotransmitter

The National Council of Scientific Development and Technology (CNPq) of the Brazilian Government provided a doctoral-SW fellowship to V.S.V. This research was supported by the National Science Foundation(NSF) Science and Technology Center for Biological Timing, by a Bristol-Myers Squibb unrestricted grant in Neuroscience to J.S.T., and by NIH Grants RO1 HL59598 to F.W.T. and P01 AG11412 to F.W.T. and J.S.T. J.S.T. is an Investigator in the Howard Hughes Medical Institute. The authors are indebted to Erik Naylor for his valuable help with data analysis and to Diego Golombek and Kathryn Scarbrough for helpful comments. Correspondence concerning this article should be addressed to D. E. Kolker, Department of Neurobiology and Physiology, Northwestern University, 2153 N. Campus Drive, Evanston, IL 60208-3525 (e-mail: dakolker@nwu.edu). binding and synthesis (Wesemann \& Weiner, 1990), hormone secretion (Van Cauter \& Turek, 1995), synaptic excitability (Barnes, McNaughton, Goddard, Douglas, \& Adamec, 1977), and even long-term potentiation (Dana \& Martinez, 1984; Harris \& Teyler, 1983). In view of this widespread rhythmicity, the efficiency in learning and memory processes might also be expected to manifest temporal components.

Indeed, several studies have documented temporal modulation of learning and memory processes. Nonassociative learning seems to be affected by time of day. Pigeons show clear habituation to sound stimulation during morning sessions, but not during evening sessions (Valentinuzzi \& Ferrari, 1997). In the marine fish Serranus scriba, avoidance and food-related learning are more effective in the evening than in the morning (Kovacevic, Rakic, \& Radil, 1991; Rakic, Kovacevic, \& Redil, 1991). In rats, performance in passive and active avoidance tasks is modulated by time of testing (Catalá, Pallardo, Roman, Villanueva, \& Viña Giner, 1985; Davies, Navaratra, \& Redfern, 1973). In addition, multiple retention deficits of active avoidancelearning (Holloway \& Wansley, 1973b) and 
passive avoidance learning (Holloway \& Wansley, 1973a, 1973b; Wansley \& Holloway, 1976), as well as one-trial appetitive learning (Wansley \& Holloway, 1975), have been well characterized. These retention deficits represent an inability of the animal to retrieve the memory of a learning experience when the training-testing delay is at non-24-h intervals and are flattened by lesions of the suprachiasmatic nucleus (the master biological clock in mammals; Stephan \& Kovacevic, 1978). Maze performance in C57BL/6Ola mice also shows a clear time-of-day effect (Hoffmann \& Balschun, 1992). Finally, in humans, psychophysiological variables (e.g., reaction time, subjective alertness), short- and long-term memory (remembering a text), and cognitive performance (calculations completed) have also been shown to change as a function of time of day (Dijk, Duffy, \& Czeisler, 1992; Guerin et al., 1991; Johnson et al., 1992; Koulack, 1997; Leconte, 1989).

Although very suggestive, these previous studies have failed to establish circadian (as opposed to state-dependent or light/dark-dependent) modulation of learning processes. In other words, there are many potential confounding factors when analyzing learning in a circadian context. The different phases of a light:dark (LD) cycle can have a confounding effect owing to different visibility during the dark versus the light phase. This can result in different abilities to learn the context. In addition, bright light inhibits or "masks" nocturnal rodents' locomotor activity (Marques \& Waterhouse, 1994), thereby interfering with several common behavioral manifestations of learning. The locomotor activity rhythm itself can also have a masking effect on learning processes, depending on the behavioral response being monitored as a measure of learning. For example, active avoidance learning is reportedly enhanced during rodents' active period, whereas passive avoidance is enhanced during the rest period (Catalá et al., 1985; Davies et al., 1973). These behaviors are obviously influenced by an animal's locomotor activity rhythm. In addition, circadian modulation of simple aspects of a task, such as baseline values of the behavior measured, reactivity to the novelty of the experimental chamber and subsequent habituation, and sensitivity to cues involved in the learning task, should be considered when weighing the evidence for the effect of circadian rhythmicity on learning and memory.

We sought to examine whether circadian modulation of different types of learning occur in mice by controlling confounding features (such as lighting) and independently measuring those factors that could not be controlled (activity, habituation, response to unconditioned stimulus). The acquisition of context- and tone-dependent fear conditioning and the extinction of the fear response were determined at two circadian phases, one in the subjective day and one in the subjective night. To bypass any masking effect of an LD cycle, we worked with animals in a skeleton photoperiod (Pittendrigh, 1965) in which a single 15 -min pulse of white light presented every $12 \mathrm{~h}$ synchronized the rest-activity cycle. Our results indicate circadian modulation of learning and/or memory in contextdependent, but not in tone-cued, fear conditioning.

\section{METHOD}

\section{Subjects}

Male C57BL/6J mice were purchased from the Jackson Laboratory (Bar Harbor, ME) at 6 weeks of age. Upon arrival, the animals were group housed (5 per cage) in the animal facility at Northwestern University for 2-3 weeks under a 12:12-h LD cycle (lights on at 05:00 central standard time). The temperature was maintained constant at $21^{\circ} \pm 2^{\circ} \mathrm{C}$. Food (Teklad) and water were available ad lib. The C57BL/6J inbred strain was chosen because they show very good performance in learning tasks, particularly on context and cued fear conditioning (Owen, Logue, Rasmussen, \& Wehner, 1997; Paylor, Tracy, Wehner, \& Rudy, 1994), and because they have robust and precise circadian rhythms of wheel-running activity (Vitaterna et al., 1994). After 2-3 weeks of acclimation, the animals were transferred to the experimental room and assigned to groups. Forty mice were randomly divided into two groups ( $n=20$ per group). One group was trained and tested in the morning. The other group was trained and tested in the evening. Each of these groups was subdivided into control and experimental groups ( $n=10$ per group).

\section{Fear Conditioning Apparatus}

The Freeze Monitor (San Diego Instruments) consisted of a transparent acrylic conditioning chamber $33 \mathrm{~cm}$ high, $25 \mathrm{~cm}$ wide, and $21 \mathrm{~cm}$ deep. A grid floor made of stainless steel rods separated by $0.5 \mathrm{~cm}$ was connected to a shock generator (Coulberg). A frame (33 $\times 33 \mathrm{~cm})$ with 16 infrared photobeams $(2.5 \mathrm{~cm}$ between beams $)$ in the horizontal plane surrounded the chamber. The conditioning chamber and surrounding frame were located inside a sound-attenuated enclosure (interior dimensions were $50 \mathrm{~cm}$ high $\times 65 \mathrm{~cm}$ wide $\times$ $47 \mathrm{~cm}$ deep). The inside of the enclosure was covered by gray acoustical foam. A small fan located on the top of the right wall generated a background noise of $65 \mathrm{~dB}$. A speaker (Mallory, SC628) was placed on the upper part of the back wall. Two night-lights on the ceiling of the chamber generated green dim light (0.5-1.0 lux at the level of the grid floor). Distinct geometric shapes of white paper were distributed on the inside walls of the sound chamber to increase the availability of visual cues.

The Freeze Monitor software (San Diego Instruments) controlled the shock generator and speaker and recorded data from the photobeams. This equipment provides an alternative measure of fear conditioning, instead of the direct observation of freezing behavior. This measure was the latency between photobeam interruptions, recorded as the latency to break the third photobeam in each 5-sec interval. If a third beam interruption never occurred, a score of $5 \mathrm{sec}$ was recorded for that interval. These latency values were added to obtain the cumulative latency (in seconds) for each minute of each session. This measure is positively correlated with freezing; thus, high latency scores reflect high amounts of freezing behavior. Other types of measures based on latency between photobeam interruptions can also be obtained with the Freeze Monitor software, each of which has been previously shown to significantly correlate $(p<$ $.0003)$ with hand-scored freezing. Correlation coefficients $(r)$ for these different computer measures ranged from .87 to .94 (see Valentinuzzi et al., 1998, for more detailed explanation and validation of this equipment and software). Latency to third beam interruption is presented here because it showed the highest correlation $(r=$ .94) with hand-scores. However, statistical analysis using the other Freeze Monitor measures indicated the same group differences and treatment effects (data not shown).

\section{Procedure}

Light cycles. Following 2-3 weeks' acclimation, the animals were transported to the experimental room, where they were placed in individual cages $(15 \times 32 \mathrm{~cm})$ with running wheels $(11 \mathrm{~cm}$ in diameter). Cages were placed in light-tight ventilated wooden cabinets $(44 \mathrm{~cm}$ high $\times 180 \mathrm{~cm}$ long $\times 53 \mathrm{~cm}$ deep), where, for the first 3 days, the conditions were the same as those in the animal facility 
(LD 12:12; light provided by 40-W "cool white" fluorescent bulb, 300 lux). After this interval, the 12-h dark phase was replaced by $12 \mathrm{~h}$ of dim (1 lux at the level of the cage lids) green light; this condition continued for two cycles. Immediately thereafter, a skeleton photoperiod (Pittendrigh, 1965), consisting of two 15-min bright white 40-W fluorescent light pulses separated by 11.5 and $12 \mathrm{~h}$ of green dim light (i.e., 0.25 white: 11.5 green:0.25 white: 12 green) was established and continued for the duration of the experiment (see Figure 1). In accordance with conventions, the end of the evening light pulse (signaling dusk) was considered zeitgeber time 12 (ZT12), and the start of the morning light pulse (signaling dawn) ZT24 (also ZT00). If synchronization to the skeleton photoperiod is adequate, activity onset should occur close to ZT12. This ZT was used as reference to determine when training and testing would occur in each group. The dim green light was provided by six night-lights (Limelite, Austin Instruments, Austin, TX) evenly distributed along the rear wall of each light-tight chamber. Green light was chosen to ensure that the mice could assess the visual cues in the chamber, since this should be an effective wavelength for mouse vision (Balkema $\&$ Pinto, 1982). This skeleton photoperiod procedure allowed testing of the animals at different circadian times, but under identical lighting conditions, therefore eliminating any exogenous component related to the LD cycle during the test.

Activity recording. Wheel-running activity was recorded continuously throughout the experiment with a 20-channel EsterlineAngus event recorder connected to the running wheels via a microswitch. The resulting activity data were displayed in actograms that were used to monitor entrainment to the skeleton photoperiod and confirm the circadian phase at which each animal was trained and tested. Only those animals exhibiting a circadian period of $24 \mathrm{~h}$, indicating entrainment to the skeleton photoperiod, were allowed to proceed in the experiment. Data from animals whose activity onset varied $30 \mathrm{~min}$ or more on 5 consecutive days were excluded from further analysis. Three mice from the night-control group, one from the night-experimental group, two from the morning-control group, and two from the morning-experimenta 1 group were excluded under this criterion.

Context fear conditioning. During the training session, the animals were placed in the conditioning chamber for $6 \mathrm{~min}$. At the beginning of minutes 4,5 , and 6 , each animal in the experimental group received a 1 -sec shock $(0.6 \mathrm{~mA})$. Control animals were handled identically but received no shock. Testing for acquisition of the conditioned response was carried out $24 \mathrm{~h}$ later. The test session consisted of placing each mouse in the enclosure for an 8-min monitoring period. After the first testing session, the animals were submitted to additional tests every $24 \mathrm{~h}$ for the next 4 days to analyze extinction of the conditioned response. After each training and testing session, the acrylic chamber was cleaned with $70 \%$ ethanol.

Tone-cued fear conditioning. Twenty-four hours after the last context fear-conditioning test, the animals were submitted to a tonecued fear-conditioning paradigm. The training session consisted of placing the animals in the conditioning chamber for $6 \mathrm{~min}$. The tone/shock pairing occurred on minutes 3,4 , and 5 and consisted of a $20-\mathrm{sec} 2.2-\mathrm{kHz}$ tone immediately followed by a $1-\mathrm{sec} 0.6-\mathrm{mA}$ shock. The animals were tested at 24 -h intervals for 5 days. Between the training and the testing sessions, the conditioning chamber was altered to avoid any expression of conditioning to the context. The walls of the environmental chamber were covered with white cardboard to alter visual cues, and the gridded floor was covered with acrylic to alter tactile cues. Before each session, the conditioning enclosure was cleaned with $1 \%$ acetic acid instead of ethanol, to alter olfactory cues. The 6-min testing sessions consisted of presenting the 20 -sec tone during the beginning of the 3rd, 4th, and 5th minutes. Control animals were exposed to the tones during both the training and testing sessions but never received shocks.

Reactivity to shock. To determine whether there was an effect of time of day on sensitivity to the shock, the same control animals were submitted to an additional 4-min session at the appropriate time points $(n=5$ mice each at ZT02 and ZT14). Three shocks ( $1 \mathrm{sec}, 0.6 \mathrm{~mA}$ ) were given at minutes 2,3 , and 4 . The behavioral

\section{Time of Day}

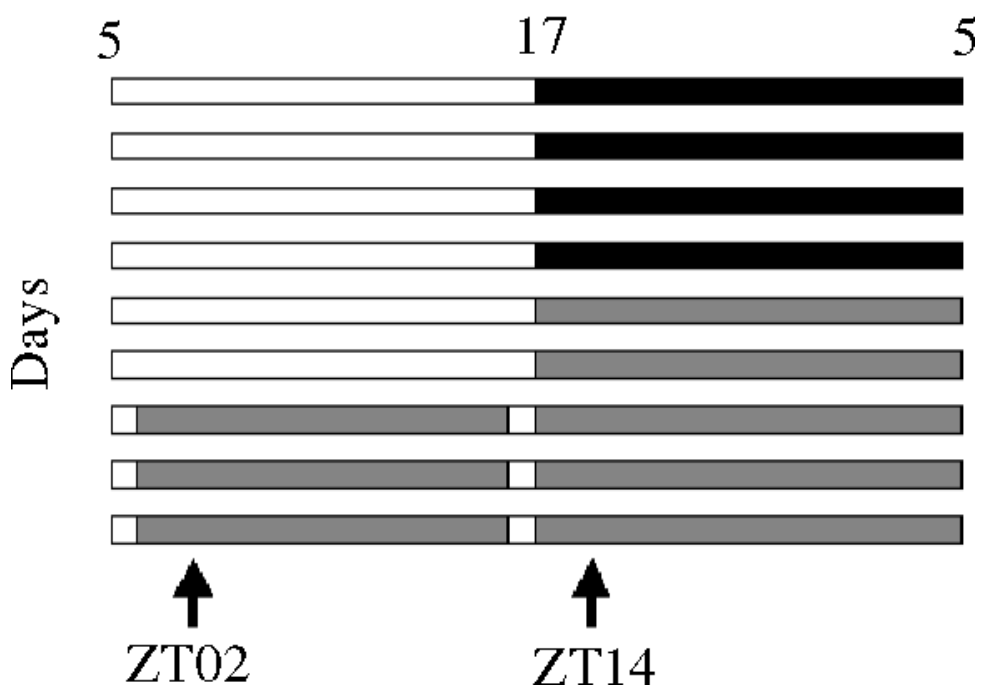

Figure 1. Schematic representation of the light:dark schedule and transfer to the skeleton photoperiod. Time is plotted across the horizontal axis (each bar represents $24 \mathrm{~h}$ ), and successive days are plotted beneath one another. White areas indicate lights-on, black areas indicate lights-off, and gray areas represent dim green light. The arrows indicate the times of testing for the ZT02 and ZT14 groups, respectively. 
response at the moment of each shock was observed and recorded by two independent observers. Interscorer reliability was determined by dividing coincident behaviors judged by the total number of observations. This reliability value between observers was high (.93). The behaviors recorded (as either occurring or not) were vocalization, running, and jumping.

\section{Analysis of Data and Statistics}

Alpha levels for all statistical tests were set at .05. Context fearconditioning results were analyzed using the mean latency/minute per session - that is, the cumulative latency of the whole session divided by the number of minutes. Tone-cued fear-conditioning sessions were analyzed in three parts. The mean latency/minute was used to analyze the first $3 \mathrm{~min}$ of the sessions before tone presentation (pretone) and the second 3 min, during which the tone was presented (tone). The amount of freezing in response to the tone was analyzed as a net tone latency for each animal — that is, the mean latency per minute during tone presentation minus the mean latency before the tone presentation (tone - pretone).

In order to analyze the effect of time of day on unconditioned components of the behavior, data from the control groups were analyzed separately. A two-way analysis of variance (ANOVA), with one grouping variable (time of day) and one repeated measure (testing sessions 1-5), was used in each fear-conditioning paradigm.

Three-way ANOVAs, with two grouping variables (time of day and experimental/control group) and one repeated measure (Testing Sessions 1-5), were performed on data from all the animals for both context fear conditioning and tone-cued fear conditioning.

In addition, in order to isolate associative components from nonassociative components, difference scores were calculated. For context fear conditioning, the difference scores were calculated as each experimental animal's latency score minus the mean latency of the appropriate control group (i.e., morning or evening and session number). Similarly, for the tone-cued fear-conditioning paradigm, difference scores were calculated as the net tone latency of each experimental animal minus the mean net tone latency of the appropriate control. These difference scores were subjected to two-way ANOVAs, with one grouping variable (time of day) and one repeated measure (Testing Sessions 1-5). For post hoc comparisons, the Tukey's HSD test was used.

Unpaired $t$ tests were used to compare the morning and the evening mice's reactivity to shock.

\section{RESULTS}

Stable entrainment to the skeleton photoperiod was confirmed by the activity records. Figures $2 \mathrm{~A}$ and $2 \mathrm{~B}$ show representative actograms of 2 well-entrained animals, one mouse trained and tested at ZT02 and another trained and tested at ZT14. During the 1st week, a clear activity rhythm synchronized to the LD cycle was observed, with activity onset occurring at the same time (lights off) every day. When transferred to the skeleton photoperiod (second arrow), activity rhythms continued to show entrainment to the external cycle. The vertical bars indicate the time of training and testing; as is depicted in Figures 2A and $2 \mathrm{~B}$, these times correspond to $2 \mathrm{~h}$ after the morning and the evening light pulses and are clearly within the inactive and the active phases, respectively. As was mentioned above, animals from each of the four groups were excluded from analysis because of the lack of stable entrainment (onset variance within 30 min over 5 days of entrainment).

Context fear conditioning. Figure 3 depicts the mean latency per minute of each context fear-conditioning session. A clear difference between the experimental and the control animals was observed [condition effect, $F(1,28)=$ 40.96] revealing that conditioning to the context occurred in the experimental animals at both ZT02 and ZT14 and across sessions. An effect of time of day on latency levels $[F(1,28)=9.51]$ and between-sessions change $[F(4,112)=15.59]$ was also evident. At both time points, the experimental animals showed high latency scores during Test 1 and a progressive decrease over the ensuing sessions. The experimental animals' latency levels and the between-sessions rate of change differed from those of the control animals and showed a time-of-day effect [time $\times$ session interaction, $F(4,112)=3.71$; condition $\times$ session interaction, $F(4,112)=22.32$; time $\times$ condition $\times$ session interaction, $F(4,112)=5.25$ ].

The experimental animals' latency scores are the combination of both associative (fear conditioning) and nonassociative (spontaneous locomotor activity, exploration of the context, as well as habituation to the environment) effects, whereas the control animals' scores reflect the nonassociative aspects alone. In order to separate these two classes of processes and thus better visualize the conditioned component, we analyzed the responses of the control animals separately. The analysis of the data from the control animals provides a baseline of the unconditioned responses: spontaneous locomotor activity, which is modulated by circadian rhythms, exploration of the novel environment, and habituation to this environment. Control animals tested at ZT02 and ZT14 differed in latency levels [time of day effect, $F(1,13)=9.92$ ]. This difference likely reflects the effect of the circadian clock on locomotor activity. As was expected, control mice placed in the testing chamber at ZT02 were less active (higher latency values) than their counterparts tested at ZT14. A session effect $[F(5,52)=4.65]$ was observed in control mice, probably reflecting some degree of habituation to the chamber. However, this habituation process was not affected by time of day (time $\times$ session interaction, n.s.).

To examine the effect of time of day on the conditioned component of the response alone, we computed the difference between the latency of the individual experimental animals and the mean value of the control group of the corresponding sessions (Figure 4). In Testing Session 1 there was a clear difference in the conditioned response between the ZT14 and ZT02 groups [time-of-day effect, $F(1,15)=8.40$ ]. The group tested during the active phase (ZT14) showed a significantly higher latency level $(27.3 \pm 2.2 \mathrm{sec} / \mathrm{min})$ than did the group tested during the inactive phase (ZT02, $15.5 \pm 1.9$, Tukey's test). Both groups showed extinction of the response [session effect, $F(4,60)=50.56]$. The rate of decrease in latency differed between groups, as is indicated by the significant time-of-day $\times$ session interaction $[F(4,60)=11.86]$. The ZT02 group reached an asymptotic latency score in the second testing session (no difference between Sessions $2-5$, Tukey's test), whereas the ZT14 group did not reach this level until the fifth testing session.

Tone-cued fear conditioning. The results from the tone-cued fear-conditioning paradigm are shown in Fig- 


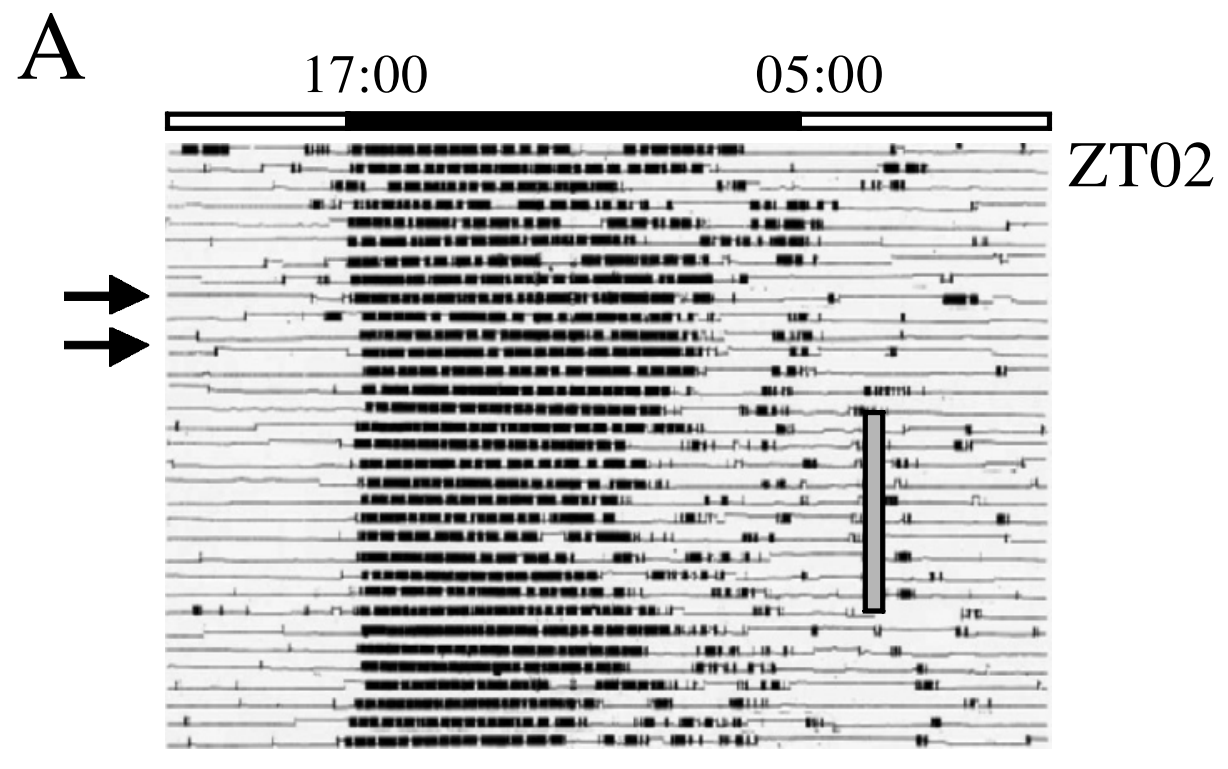

B

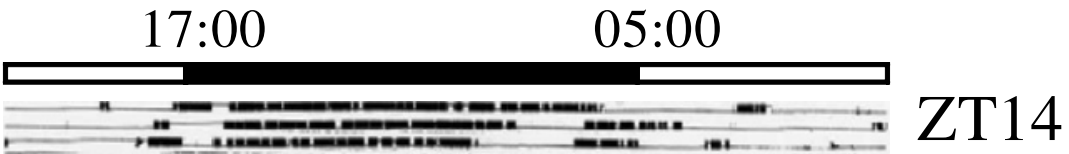

Figure 2 (A) Representative actogram of an animal trained and tested at ZTO2. (B) Representative actogram of an animal submitted to fear conditioning at ZT014. Time is plotted across the horizontal axis ( $24 \mathrm{~h}$ per line), and successive days are plotted beneath one another. The bar on the top indicates the light:dark cycle during the first week. The first arrow indicates when the dark phase was replaced by green dim light, and the second one indicates when the skeleton photoperiod started. The vertical gray bars indicate when training and testing for both context and tone-cued fear conditioning occurred.

ure 5, where they are expressed as mean latency/minute of the testing sessions before the tone is presented (Figure 5, top panel) and during presentation of the tone (Figure 5, middle panel). The pretone part of the session gives information on the unconditioned components mentioned previously, as well as any residual conditioning to the context that is visible as the difference between experimentals and controls. The three-way ANOVA of data from the pretone part of the session revealed a significant main effect of time of day $[F(1,28)=17.32]$; this effect is best explained by the circadian rhythm of locomotor activity. As was observed in the control animals during context fear-conditioning sessions, ZT02 animals showed higher latency values than did ZT14 animals. The ANOVA of the data from this part of the session also revealed a condition effect $[F(1,28)=18.00]$, indicating that the 


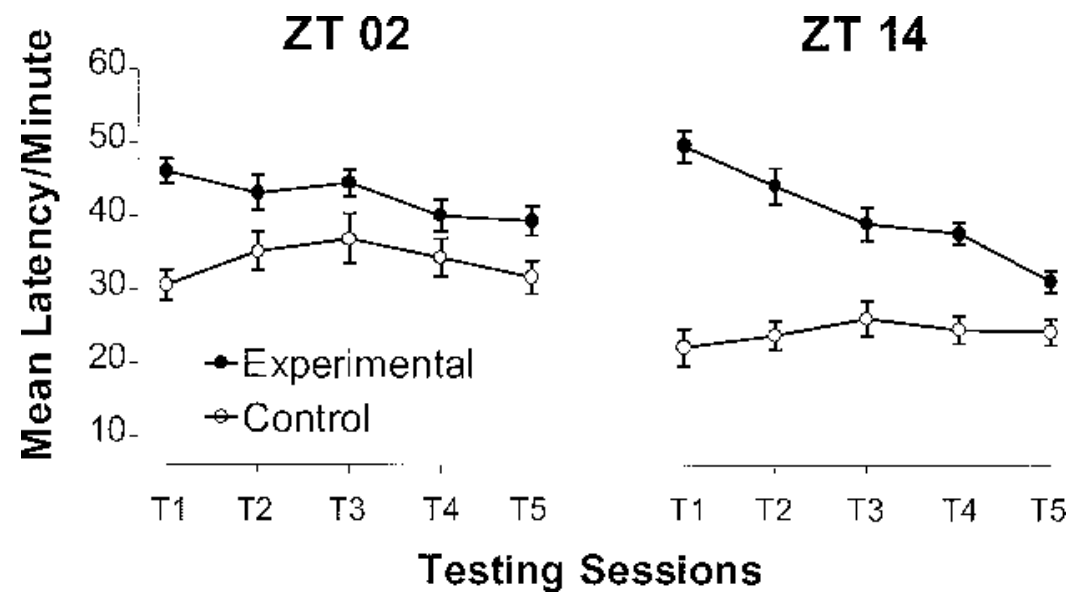

Figure 3. Mean $\pm S E M$ latency per minute for each of the five context-conditioning testing sessions of the control group (open circles) and the experimental group (black circles). The left panel shows the animals tested during their inactive phase (ZT02), and the right panel shows the animals tested during their active phase (ZT14).

experimental animals showed residual conditioning to the chamber despite its alteration. No time $\times$ condition $X$ session interaction was detected before presentation of the tone. When the tone was presented in the second half of the session (Figure 5, middle panel), freezing increased significantly more in the experimental animals than in the control animals [main effect of condition, $F(1,28)=$ 179.61], but no effect of time of day was detected. None of the other main effects or interactions was significant. However, it is the relative increase in freezing that will give us information on the associative gain - that is, the response to the tone relative to the latency level before the tone is presented. By calculating each animal's net tone latency, we can control for individual differences in

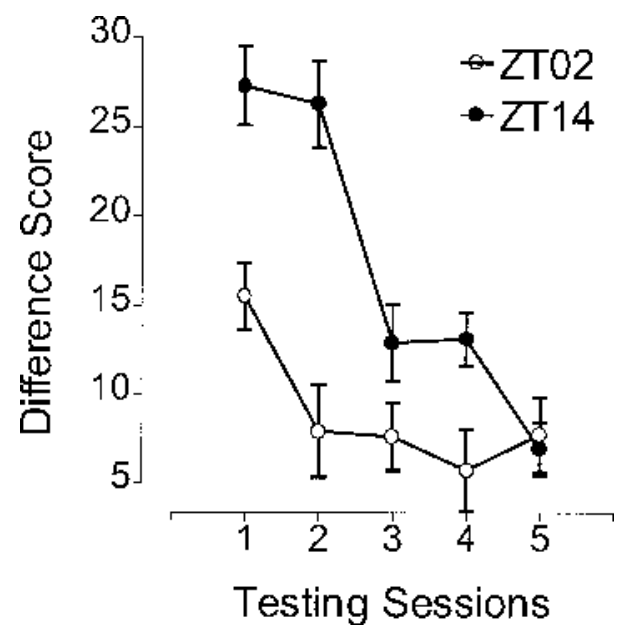

Figure 4. Mean $\pm S E M$ latency per minute for each testing session of the experimental animals tested for context fear conditioning at ZT14 (black circles) and ZTO2 (open circles) after subtracting the appropriate control values (difference scores; see the Method section). exploration and habituation to the context, rhythm of locomotor activity, as well as any previous conditioning to the chamber, effectively isolating the response to the tone. These net tone latency values are shown in the lower panel of Figure 5.

The analysis of the net tone latency of both the experimental and the control animals permits a comparison between the conditioned and the unconditioned responses to the tone. There was a significant main effect of condition $[F(1,28)=67.51]$, revealing that conditioning to the tone occurred in the experimental animals at both time points and across the testing sessions. In addition, both the control and the experimental animals showed a decrease in the net tone values from session to session at both time points [session effect, $F(4,112)=9.34$ ], probably owing to the increases in latency during the pretone period (top panels), since latency to the tone appears unchanged across test sessions (middle panel). And finally, a significant effect of time of day $[F(1,28)=7.09]$ on net tone latency was also observed. This is best explained by the temporal effect on sensitivity to the tone detected in the control animals, because the changes observed in the experimental animals are also present in the control animals at both time points. However, the lack of significant effects in any of the time-of-day interactions indicates the absence of a temporal modulation of conditioning and extinction to a tone (the time $\times$ condition, the time $\times$ session, and the time $\times$ condition $\times$ session interactions were not significant).

In addition, the analysis of data from the unshocked control animals provides a way to evaluate sensitivity to the tone and subsequent habituation to it. The ANOVA revealed a significant main effect of time of day $[F(1,13)=$ $5.10]$ on net tone latency in control animals; these values were lower in the ZT02 control animals than in the ZT14 control animals. This suggests that control animals exposed to a tone at ZT14 respond to it with more inactiv- 


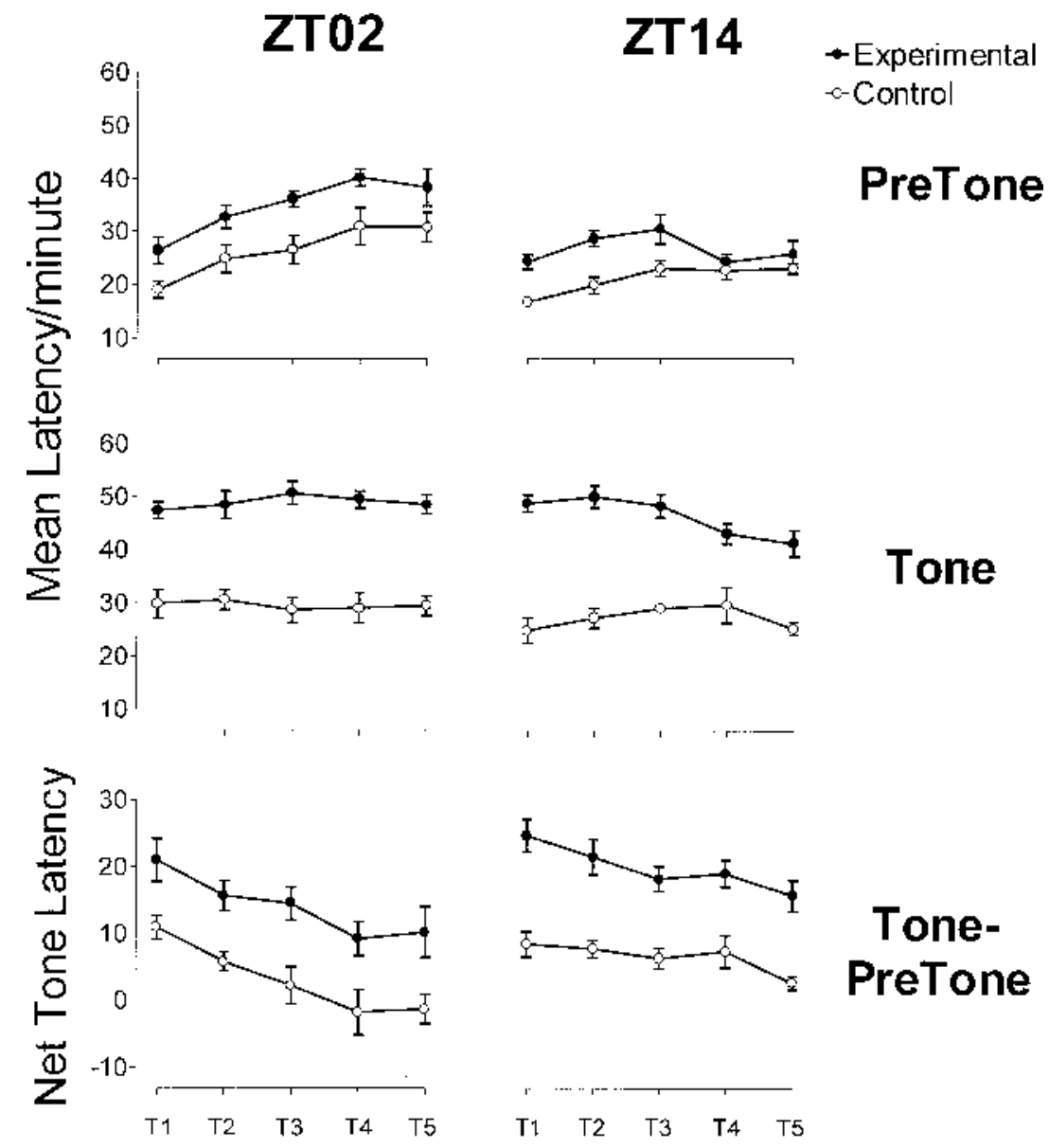

\section{Testing Sessions}

Figure 5. Mean $\pm S E M$ latency per minute for each of the five context-conditioning testing sessions of the control group (open circles) and the experimental group (black circles). The left panels show the animals tested during their inactive phase (ZT02), and the right panels show the animals tested during their active phase (ZT14). The top panel shows the first $3 \mathrm{~min}$ of the sessions before tone presentation (pretone). The middle panel shows the second half of the sessions, during which the tone was presented (tone). The bottom panel shows the latency values during tone presentation after subtracting the pretone baseline.

ity (or more freezing), even though they have a greater drive from the circadian timing system to be active. A decrease in latency levels across sessions was also observed $[F(5,52)=5.53]$, indicating habituation to the tone. However, no time-of-day difference was evident in this habituation process (time $\times$ session interaction, n.s.).

We also tried to dissect the unconditioned (reactivity to the novelty of the tone, as well as habituation to it) and the conditioned (conditioning to the tone) components of tone-cued fear conditioning. To do this, we subtracted the mean net tone latency of the appropriate (ZT02 or ZT14) control groups from each experimental animal's net tone latency. These difference scores are depicted in Figure 6. No significant main effect or interaction was detected between the groups on this measure.
Reactivity to the shock. To determine whether there was an effect of time of day on perception of the unconditioned stimulus, we quantified the immediate behavioral responses to the shock. The type and level of these responses were the same at both time points. Unpaired $t$ tests revealed equal vocalization, jumping, and running responses. At both times of day, running was the most common response to the shock.

\section{DISCUSSION}

The present data show clear temporal modulation of complex learning or memory processes when typical time-of-day confounds, such as the LD cycle, are controlled. Expression of context-dependent fear condition- 


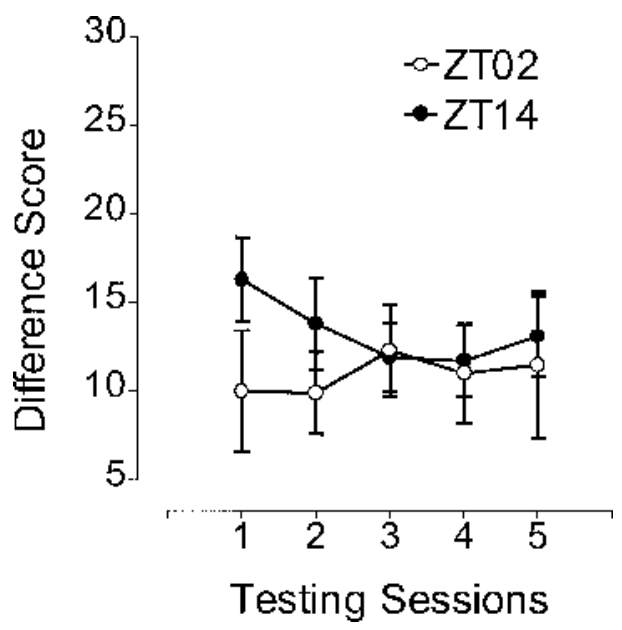

Figure 6. Mean $\pm S E M$ latency per minute for each testing session of the experimental animals tested for tone-cued fear conditioning at ZT14 (black circles) and ZT02 (open circles) after extracting the control values (difference scores; see the Method section).

ing, which is thought to be hippocampal dependent, was more pronounced, and the rate of extinction was faster among mice tested during their active phase than among those tested during their inactive phase. In contrast, tonecued conditioning, a presumed hippocampal-independent learning process (Paylor et al., 1994; Phillips \& Le Doux, 1992), was not influenced by time of day.

During context fear-conditioning sessions, data from control animals enabled us to quantify the unconditioned responses. The significantly different latency levels between the morning and the evening control groups are best explained by the circadian rhythm of locomotor activity. Presumably because they have an increased drive to be active from the circadian clock, control animals tested during their active period show lower latency values than do those tested during their rest period. Another phenomenon expected to be observed in these control animals was habituation to the context. Rodents placed in a novel environment will characteristically show an initial high level of activity that decreases over time (Cerbone \& Sadile, 1994). However, this pattern was not obvious at either time point. Thus, circadian differences in activity level did result in differences in the unconditioned response.

The conditioned component of the behavior during testing is reflected by the difference between the experimental and the control curves. This is more pronounced at ZT14, suggesting that evening animals show better performance in this conditioning task than do morning animals. If the observed difference in freezing levels were controlled entirely by the circadian rhythm of locomotor activity, one would expect that the animals trained and tested during the inactive phase (ZT02) would have higher latency scores than the animals trained and tested during the active phase (ZT14). However, our data show the opposite results: Experimental animals tested during the active phase expressed higher latency values (more freezing behavior) than did experimental animals tested during ZT02, thereby ruling out a facilitating effect of the inactive phase on freezing. Thus, the effect of time of day that we observe on freezing behavior is better explained by differences in learning or memory processes. In addition, if all the time-of-day effects on freezing were driven by the circadian rhythm of locomotor activity, one would expect to see such differences in tone-cued fear conditioning as well. However, such an effect is not apparent, suggesting that activity rhythms and fear-conditioning responses are independent processes.

We also sought to examine the associative-learning component by calculating difference scores. This procedure seemed to be effective in extracting a time-of-day effect on the conditioned component of the behavior (see Figure 4). This analysis assumed that the associative and nonassociative components of the behavioral response are simply additive. However, using difference scores appears to be a reasonable way to control for the different baseline activity, and other authors have used similar procedures (Paylor et al., 1994). Also, the fact that the intersubject variability in our control animals was very small increases the utility of this type of analysis. When an analysis that assumed the effects were multiplicative was used (i.e., when the ratios of the experimental to control latency values was used), as well as a matched-pair approach (based on basal activity levels between the experimental and the control animals), the same time-ofday differences were detected. Thus, it appears that the associative-learning processes (conditioning to the context) are affected by time of day.

In contrast with context fear conditioning, tone-cued fear conditioning appears to be independent of circadian phase (Figure 6). However, other components of the behavioral assay are affected by time of day, and these had to be controlled in order to isolate fear conditioning to the tone. As with context conditioning, the latency measures included the effects of the activity rhythm and habituation to the context and are visible in the first half of the tone-cued testing sessions. The first part of the session also revealed residual conditioning responses to the context owing to the previous experience of these animals. The experimental groups tested at ZT02 and ZT14 showed different levels of reactivity to the tone when net tone latency was evaluated (see the lower panel of Figure 5), but our analysis suggests that this can be explained by nonassociative components of the response to the tone, since the difference seen between ZT02 and ZT14 experimental animals in response to the tone is also seen to the same extent in control animals. Finally, the control animals' decrease in the net tone latency across sessions, which indicates habituation to the tone, was independent of time of day. When these other factors are taken into consideration, as we have attempted to do in calculating the difference scores, no time-of-day effects in cued conditioning are apparent.

There are two potential confounding factors in our experimental design that should be discussed. One is the 
interpretation flaw created by the differences in baseline responding as seen in the control groups. We cannot rule out the possibility that the greater unconditioned freezing in the group tested at ZT02 could mask associative effects or, conversely, that the greater ambulation by active mice unmasked learning. However, we think this is not the case, since this type of masking effect would presumably be present in tone-cued fear conditioning and is not apparent. Although this may be resolved by additional groups trained at one ZT but tested at another, this would result in comparing animals with different training-testing intervals, which can lead to different performances (Holloway \& Wansley, 1973a, 1973b; Wansley \& Holloway, 1975, 1976).

The other limitation is that the context fear-conditioning procedure could have had carryover effects on tone-cued fear conditioning. Although some carryover effect does exist, the pretone data suggest that it is not an effect specific to time of day. Had greater context conditioning carried over at ZT14, greater freezing should have manifested itself to residual contextual cues during tone testing at ZT14; just the opposite appears to be true. The pretone data are evidence against differential carryover effects between groups as they progress from context to tonecued conditioning.

The differences in circadian modulation of contextand cue-dependent conditioning may arise from the distinct neuroanatomical pathways underlying these two types of conditioning. The amygdala is involved in the formation of associations between aversive unconditioned stimuli and a variety of conditioned stimuli types, as well as in the subsequent control of a fear response (Maren \& Fanselow, 1996). However, different neural pathways mediate the analysis of the stimulus properties, depending on the characteristics of the specific stimuli involved in the situation (Kim \& Fanselow, 1992; Paylor et al., 1994; Phillips \& LeDoux, 1992, 1994). For modalityspecific conditioned stimuli, either thalamic or cortical inputs to the amygdala suffice as transmission routes. For more complex stimuli, such as those that involve spatial organization, the hippocampus, as part of its general role in spatial processing, and the projection from the subiculum to the amygdala are required (Eichenbaum, Otto, \& Cohen, 1992). The participation of a complex and integrative structure such as the hippocampus (Eichenbaum et al., 1992) in context-dependent fear conditioning, but not in tone-cued fear conditioning, may lead to increased susceptibility of contextual conditioning to external modulation. Indeed, a number of variables influence contextual, but not cue-dependent, fear conditioning. Postconditioning isolation (Rudy, 1996), retention interval (Rudy \& Morledge, 1994), stimulus preexposure (Rudy, 1996; Rudy \& Morledge, 1994), and age (Rudy, 1993) affect conditioning to a context. This pattern suggests a vulnerability in the consolidation process of the context characteristics. Our data add time of day as another possible modulatory variable.

It is tempting to hypothesize that the biological clock could be directly or indirectly affecting the hippocampal complex; several hippocampal variables manifest rhythmicity and thus are potential mechanisms for such clock influences. The expression of glucocorticoid and mineralcorticoid receptor mRNA (Holmes et al., 1995), as well as 5-HT2C receptor mRNA expression (Holmes, French, \& Seckl, 1997), shows diurnal rhythms. The hippocampus also expresses rhythmicity in Jun-B and c-fos mRNA (Menegazzi et al., 1994) and in Fos protein levels (Kononen et al., 1990). Plasma corticosterone level, which is itself driven by the circadian clock (Van Cauter \& Turek, 1995), is known to have a significant effect on cognition, mainly by acting on the hippocampus (Lupien \& McEwen, 1997). Corticosterone levels affect activation of kainate receptors (Joels, Bosma, Hendriksen, Diegenbach, \& Kamphius, 1996) and mRNA levels of $\mathrm{GABA}_{\mathrm{A}}$ receptor subunits (Orchinik, Weiland \& McEwen, 1994), consequently affecting hippocampus excitability. Circadian rhythms in synaptic excitability (Barnes et al., 1977) and long-term potentiation (Dana \& Martinez, 1984; Harris $\&$ Teyler, 1983) have been detected in the rat and the monkey hippocampus. It will be of interest to determine which, if any, of these hippocampal rhythms underlie modulation of contextual learning and memory.

The adaptive value, if any, of having temporal modulation of context fear conditioning, but not of tone-cued fear conditioning, remains unclear. Discrete stimuli may be perceived more as an imminent danger that can appear at any time and at any place. An animal cannot afford to have its ability to associate such stimuli with danger reduced at a particular time of day. On the other hand, learning a context may be more related to foraging, mating, and establishing territory. These are all activities that take place during the active phase. A decreased capacity to learn contextual cues during the inactive phase may not be much of a disadvantage to an animal in a well-known context, its nest.

\section{REFERENCES}

Balkema, G. W., \& Pinto, L. H. (1982). Electrophysiology of retinal ganglion cells in the mouse: A study of a normally pigmented mouse and a congenic hypopigmentation mutant, pearl. Journal of Neurophysiology, 48, 968-980.

Barnes, C. A., McNaughton, B. L., Goddard, G., Douglas, R. M., \& Adamec, K. (1977). Circadian rhythm of synaptic excitability in rat and monkey CNS. Science, 197, 91-92.

Catalá, M. D., Pallardo, F., Roman, A., Villanueva, P., \& Viña GiNER, J. M. (1985). Effect of pinealectomy and circadian rhythm on avoidance behavior in the male rat. Physiology \& Behavior, 34, 327-333.

Cerbone, A., \& SAdile, A. G. (1994). Behavioral habituation to spatial novelty: Interference and noninterference studies. Neuroscience \& Biobehavioral Reviews, 18, 497-518.

Dana, R. C., \& Martinez, J. L. (1984). Effect of adrenalectomy on the circadian rhythm of LTP. Brain Research, 308, 392-395.

Davies, J. A., Navaratra, V., \& Redfern, P. H. (1973). A 24-hour rhythm in passive-avoidance behavior in rats. Psychophysiology, 32, 211-214.

Dijk, D. J., Duffy, J. F., \& Czeisler, C. A. (1992). Circadian and sleep/wake dependent aspects of subjective alertness and cognitive performance. Journal of Sleep Research, 1, 112-117.

Eichenbaum, H., Otto, T., \& Cohen, N. J. (1992). The hippocampus: What does it do? Behavioral \& Neural Biology, 57, 2-36.

Guerin, N., Boulenguiez, S., Reinberg, A., Di Costanzo, G., 
Guran, P., \& Tourtou, Y. (1991). Diurnal changes in psychophysiological variables of school girls: Comparison with regard to age and teacher's appreciation of learning. Chronobiology International, $\mathbf{8}$, 131-148.

Harris, K. M., \& Teyler, T. J. (1983). Age differences in a circadian influence on hippocampal LTP. Brain Research, 261, 69-73.

HofFMANN, H. J., \& BALSCHUN, D. (1992). Circadian differences in maze performance of C57BL/6 mice. Behavioral Processes, 27, 77-84.

Holmes, M. C., French, K. L., \& Seckl, J. R. (1995). Modulation of serotonin and corticosteroid receptor gene expression in the rat hippocampus with circadian rhythm and stress. Brain Research: Molecular Brain Research, 28, 186-192.

Holmes, M. C., French, K. L., \& Seckl, J. R. (1997). Disregulation of diurnal rhythms of serotonin 5-HT2C and corticosteroid receptor gene expression in the hippocampus with food restriction and glucocorticoids. Journal of Neuroscience, 17, 4056-4065.

Holloway, F. A., \& WANSLey, R. (1973a). Multiphasic retention deficits at periodic intervals after passive-avoidance learning. Science, 180, 208-210.

Holloway, F. A., \& Wansley, R. (1973b). Multiple retention deficits at periodic intervals after active and passive avoidance. Behavioral Biology, 9, 1-14.

Joels, M., Bosma, A., Hendriksen, H., Diegenbach, P., \& KamPHIUS, W. (1996). Corticosteroid actions on the expression of kainate receptor subunit mRNA in rat hippocampus. Brain Research: Molecular Brain Research, 37, 15-20.

Johnson, M. P., Duffy, J. F., Dijk, D. J., Ronda, J. M., Dyal, C. M., \& Czeisler, C. A. (1992). Short-term memory, alertness and performance: A reappraisal of their relationship to body temperature. Journal of Sleep Research, 1, 24-29.

KIM, J. J., \& FANSELOW, M. S. (1992). Modality-specific retrograde amnesia of fear. Science, 256, 675-677.

Kononen, J., Koistinaho, J., \& AlHo, H. (1990). Circadian rhythm in c-fos-like immunoreactivity in the rat brain. Neuroscience Letters, 120, 105-108.

Koulack, D. (1997). Recognition memory, circadian rhythms, and sleep. Perceptual \& Motor Skills, 85, 99-104.

Kovacevic, N., Rakic, L., \& RadiL, T. (1991). Avoidance learning in the marine fish Serranus scriba influenced by circadian rhythmicity. Homeostasis, 33, 152-153.

LeConte, P. (1989). Chronobiological rhythm constraints of memory processes. Archives of Gerontology \& Geriatrics, 1, 21-25.

LupIEN, S. J., \& McEwEN, B. S. (1997). The acute effects of corticosteroids on cognition: Integration of animal and human model studies. Brain Research Reviews, 24, 1-27.

Maren, S., \& Fanselow, M. S. (1996). The amygdala and fear conditioning: Has the nut been cracked? Neuron, 16, 237-240.

Marques, M. D., \& Waterhouse, J. M. (1994). Masking and the evolution of circadian rhythmicity. ChronobiologyInternational, 11, 46-55.

Menegazzi, M., Carcereri De Prati, A. C., \& Zucconi, G. G. (1994). Differential expression pattern of Jun B and c-jun in the rat brain during the 24-h cycle. Neuroscience Letters, 182, 295-298.

Orchinik, M., Weiland, N. G., \& McEwen, B. S. (1994). Adrenalectomy selectively regulates $\mathrm{GABA}_{\mathrm{A}}$ receptor subunit expression in the hippocampus. Molecular \& Cellular Neuroscience, 5, 451-458.

Owen, E. H., Logue, S. F., Rasmussen, D. L., \& Wehner, J. M. (1997). Assessment of learning by the Morris water task and fear conditioning in inbred mouse strains and F1 hybrids: Implications of genetics back- ground for single gene mutations and quantitative trait loci analyses. Neuroscience, 80, 1087-1099.

Paylor, R, Tracy, R, Wehner, J. M., \& Rudy, J. W. (1994). DBA/2 and C57BL/6 mice differ in contextual fear conditioning but not auditory fear conditioning. Behavioral Neuroscience, 108, 1-8.

Phillips, R. G., \& Le Doux, J. E. (1992). Differential contribution of amygdala and hippocampus to cued and contextual fear conditioning. Behavioral Neuroscience, 106, 274-285.

Phillips, R. G., \& LeDoux, J. E. (1994). Lesions of the dorsal hippocampal formation interfere with background but not foreground contextual fear conditioning. Learning \& Memory, 1, 34-44.

PITTENDRIGH, C. S. (1965). On the mechanism of entrainment of circadian rhythms by light cycles. In J. Ascholl (Ed.), Circadian clocks (pp. 277-297). Amsterdam: North-Holland.

RaKIC, L., Kovacevic, N., \& RAdIL, T. (1991). Alimentary learning in the marine fish Serranus scriba influenced by circadian rhythmicity. Homeostasis, 33, 153-154.

Rudy, J. W. (1993). Contextual and auditory cue conditioning dissociate during development. Behavioral Neuroscience, 107, 887-891.

RUDY, J. W. (1996). Postconditioning isolation disrupts contextual conditioning: An experimental analysis. Behavioral Neuroscience, 110, 238-246.

Rudy, J. W., \& Morledge, P. (1994). Ontogeny of contextual fear conditioning: Implications for consolidation, infantile amnesia, and hippocampal system function. Behavioral Neuroscience, 108, 227-234.

Stephan, F. K., \& Kovacevic, N. S. (1978). Multiple retention deficit in passive avoidance in rats is eliminated by suprachiasmatic lesions. Behavioral Biology, 22, 456-462.

TureK, F. W. (1998). Circadian rhythms. Hormonal Research, 49, 109113.

Valentinuzzi, V. S., \& Ferrari, E. A. M. (1997). Habituation to sound during morning and night sessions in pigeons (Columba livia). Physiology \& Behavior, 62, 1203-1209.

Valentinuzzi, V. S., Kolker, D. E., Vitaterna, M. H., Shimomura, K., Whiteley, A., Low-Zeddies, S., Turek, F. W., Ferrari, E. A. M., PAYlor, R., \& TAKahashi, J. S. (1998). Automated measurement of mouse freezing behavior and its use for quantitative trait locus analysis of contextual fear conditioning in (BALB/cJ X C57BL/J)F2 mice. Learning \& Memory, 5, 391-403.

VAN CAUTER, E., \& TUREK, W. F. (1995). Endocrine and other biological rhythms. In L. I. De Groot (Ed.), Textbook of endocrinology (3rd ed., pp. 2487-2548). Philadelphia: Saunders.

Vitaterna, M. H., King, D. P., Chang, A. M., Kornhauser, J. M., Lowrey, P. L., McDonald, J. D., Dove, W. F., Pinto, L. H., TureK, F. W., \& TAKAHASHI, J. S. (1994). Mutagenesis and mapping of a mouse gene Clock, essential for circadian behavior. Science, 264, 719-725.

WANSLEy, R. A., \& HollowAY, F. A. (1975). Multiple retention deficits following one-trial appetitive training. Behavioral Biology, 14, 135-149.

Wansley, R. A., \& Holloway, F. A. (1976). Oscillations in retention performance after passive-avoidance training. Learning \& Motivation, 7, 296-302.

Wesemann, W., \& Weiner, N. (1990). Circadian rhythm of serotonin binding in rat brain. Progress in Neurobiology, 35, 405-428.

(Manuscript received January 14, 2000; revision accepted for publication December 12, 2000.) 